\title{
Hybrid Coded Replication in LoRA Networks
}

\author{
Jean Michel de Souza Sant'Ana, Arliones Hoeller, Richard Demo Souza, \\ Samuel Montejo-Sánchez, Hirley Alves, and Mario de Noronha Neto
}

\begin{abstract}
Low Power Wide Area Networks (LPWAN) are wireless connectivity solutions for Internet-of-Things (IoT) applications, including industrial automation. Among the several LPWAN technologies, LORAWAN has been extensively addressed by the research community and the industry. However, the reliability and scalability of LORAWAN are still uncertain. One of the techniques to increase the reliability of LORAWAN is message replication, which exploits time diversity. This paper proposes a novel hybrid coded message replication scheme that interleaves simple repetition and a recently proposed coded replication method. We analyze the optimization of the proposed scheme under minimum reliability requirements and show that it enhances the network performance without requiring additional transmit power compared to the competing replication techniques.
\end{abstract}

Keywords-Industrial Internet-of-Things, LoRaWAN, Message Replication, Reliability, Wireless Networks.

\section{INTRODUCTION}

Many Internet-of-Things (IoT) applications [1], [2] require wireless coverage of high-density areas using low power devices with long battery lifetime. Such demands make shortrange technologies, like Bluetooth and ZigBee, as well as established cellular networks, like GSM and LTE, unfeasible solutions for different reasons, including cost, energy consumption, and limited coverage. Low Power Wide Area Network (LPWAN) technologies like LoRAWAN, SigFox, and NB-IoT [3], are natural candidates for such applications due to their long-range, large-capacity, and power efficiency [4].

Moreover, very low bit rate wide area networks (VLBR WAN), such as those built based on LPWAN technologies,

Jean Michel de Sousa Sant'Ana, Arliones Hoeller and Hirley Alves are with Centre for Wireless Communications, University of Oulu, Oulu, Finland ( $\{$ Jean.DeSouzaSantana, Hirley.Alves $\} @$ oulu.fi).

Arliones Hoeller and Mario de Noronha Neto are with the Department of Telecommunications, Federal Institute for Education, Science and Technology of Santa Catarina, São José, Brazil (\{arliones.hoeller, noronha\} @ifsc.edu.br)

Richard Demo Souza and Arliones Hoeller are with the Department of Electrical and Electronics Engineering, Federal University of Santa Catarina, Florianóplis, Brazil (richard.demo@ufsc.br)

Samuel Montejo-Sánchez is with Programa Institucional de Fomento a la I+D+i, Universidad Tecnológica Metropolitana, Santiago, Chile. (smontejo@utem.cl)

This work has been partially supported in Brazil by CNPq, FUMDESUNIEDU, Print CAPES-UFSC Project Automation 4.0, and INESC Brazil Project F-LOCO (Energisa/ANEEL PD-00405-1804/2018); in Finland by Academy of Finland (Aka) 6Genesis Flagship (Grant 318927), EE-IoT (Grant 319008), Aka Prof (Grant 307492), and FIREMAN (Grant 326301); and in Chile by FONDECYT Postdoctoral (Grant 3170021). are viable wireless solutions for several Industrial IoT applications demanding the coverage of several devices, with latency and reliability constraints that are somewhat milder for the industrial scenario. Among these applications, we may cite [5]: monitoring and supervisory control of flow-based systems; factory monitoring of job-based systems; tracking of personnel, tools, and materials; as well as machinery health monitoring.

Among the LPWAN technologies, LoRAWAN network protocols [6], based on LoRA physical layer technology [7], is attracting much attention not only from the academy but also from industry [8]. However, the ultimate performance limits of LORAWAN are still unknown, while many open questions remain on how to increase or even to guarantee the reliability demanded by typical industrial applications. This paper focuses on the capacity of LORAWAN in terms of the number of devices in coverage, while meeting a given target reliability constraint, and when using message replication techniques to exploit time diversity. We build on the repetition replication scheme investigated in [9] and on a coded replication scheme recently proposed in [10], to introduce a novel hybrid replication approach optimized for LORAWAN, which can increase the network performance with respect to [9] and [10].

\section{A. Related Work}

Georgiou and Raza [11] present a mathematical model taking into account connection and collision probabilities to analyze large-scale LORA networks. They show that collisions are the most limiting factor. Mahmood et al. [12] present a similar model that considers accumulated interference rather than only the highest interference as in [11], besides inter$\mathrm{SF}$ interference. They show that considering the strongest colliding node is a good approximation only for low-density scenarios. Mikhaylov et al. [13] present a scalability analysis of LORAWAN, optimizing the number of nodes per SF.

There are several studies about the performance of LPWAN technologies in industrial scenarios. Sommer et al. [14] discuss and evaluate several LPWAN protocols for indoor industrial scenarios. They conclude that LoRAWAN provides the best link budget and reliability. Rizzi et al. [15] propose a time slot channel hopping scheme for LORAWAN in industrial scenarios. They show that their scheme increases network capacity to support up to 6,000 devices with the highest transmission period without collisions, if it uses all time slots. They conclude that with the proposed technique LORAWAN is compatible with the industrial requirements and similar to solutions as WirelessHART and ISA100.11a. Muzammir et al. [16] studies the feasibility of LORAWAN for indoor applications, taking into account packet losses, data rates, and communication range. They conclude that LORAWAN is 
suitable for indoor applications and encourage future works on scalability and reliability. Haxhibeqiri et al. [17] tested LoRA technology coverage in an industrial indoor area of 34,000 $\mathrm{m}^{2}$. With a simulation analysis using one gateway, all $6 \mathrm{SF}$ and 3 different frequency channels, they could support up to 6,000 devices with a success probability of 0.9 . Finally, Lentz et al. [18] present a industrial real-time monitoring system operating with LoRA technology, with sensors on a foodprocessing plant for preventive maintenance.

In a different line of research, Hoeller et al. [9] extend the model in [11] by adding spatial and time diversity, concluding that simple message replication (RT) is often beneficial but may flood the network. Mo et al. [19] investigate the same replication scheme but in SIGFOX, showing that there is an optimal number of replications. Marcelis et al. [20] present DaRe, an application layer coding technique for LoRAWAN. It features characteristics of the fountain and convolutional coding, where packets carry redundant information from previous packets. The technique improves LoRAWAN in exchange for latency. Sandell and Raza [21] analyze DaRe and show that latency increases exponentially with packet loss. Finally, Sánchez et al. [10] present some coding schemes for LPWAN. They propose Coded Transmission - Independent (CT), which presents a better success rate than the methods in [9] and [20] for several scenarios. However, none of these works analyze the energy consumption impact of such replication schemes.

\section{B. Contribution}

We present a novel coded message replication scheme, Hybrid Transmission (HT), that generalizes RT [9] and CT [10] and allows fine-tuning for different network densities and reliability targets. Moreover, in order to analyze the maximum number of devices, we numerically optimize the parameters of the proposed coded replication scheme. The new method provides important gains in terms of users in coverage compared to the methods in [9] and [10], while the advantage of the proposed scheme increases with the reliability target. On top of that, we build upon an energy consumption model [22] to analyze the impact of replications on battery lifetime. Finally, we propose a slight change to LoRAWAN to avoid unnecessary energy waste with replication schemes.

The rest of this paper is organized as follows. Section II describes LoRA and LoRAWAN. Section III presents the adopted system model. Section IV discusses some replication schemes for LPWAN. Section V introduces and analyzes the novel HT coded replication scheme. Section VI presents numerical results. Finally, Section VII concludes the paper.

\section{LORA OVERVIEW}

LORA is a proprietary sub-GHz PHY technology for longrange low-power communications that employs chirp spread spectrum. LoRA uses six practically orthogonal SFs, which allows for a rate and range tradeoff [7]. LORAWAN is the most popular network protocol using LORA as a PHY, being regulated by LoRA Alliance [6]. LORAWAN presents a star topology where devices communicate in a single-hop with the gateway, which in turn has a standard IP connection with a
Table I. LORA UPLinK CHARACTERISTICS. PAYLOAD OF 9 BYTES, $B=125 \mathrm{KHz}$, CRC AND HEADER MODES ENABLED.

\begin{tabular}{lcccc}
\hline SF & Time-on-Air $t_{j}$ & $T_{r x 1 w}$ & $T_{r x 2 w}$ & SNR threshold $q_{j}$ \\
\hline 7 & $41.22 \mathrm{~ms}$ & $12.29 \mathrm{~ms}$ & $1.28 \mathrm{~ms}$ & $-6 \mathrm{~dB}$ \\
8 & $72.19 \mathrm{~ms}$ & $24.58 \mathrm{~ms}$ & $2.30 \mathrm{~ms}$ & $-9 \mathrm{~dB}$ \\
9 & $144.38 \mathrm{~ms}$ & $49.15 \mathrm{~ms}$ & $4.35 \mathrm{~ms}$ & $-12 \mathrm{~dB}$ \\
10 & $247.81 \mathrm{~ms}$ & $98.30 \mathrm{~ms}$ & $8.45 \mathrm{~ms}$ & $-15 \mathrm{~dB}$ \\
11 & $495.62 \mathrm{~ms}$ & $131.07 \mathrm{~ms}$ & $16.64 \mathrm{~ms}$ & $-17.5 \mathrm{~dB}$ \\
12 & $991.23 \mathrm{~ms}$ & $262.14 \mathrm{~ms}$ & $33.02 \mathrm{~ms}$ & $-20 \mathrm{~dB}$ \\
\hline
\end{tabular}

network server. LORAWAN uses ALOHA [23] as medium access control, exploiting LoRA characteristics, enabling multiple devices to communicate at the same time using different SFs.

LORAWAN provides several configurations, limited by regional regulations, and based on SF, that varies from 7 to 12 , and bandwidth $(B)$, usually $125 \mathrm{kHz}$ or $250 \mathrm{kHz}$ for uplink. Higher SFs extend the symbol duration, reducing the rate and, thus, increasing robustness. Features that reduce the rate, such as higher SF and lower bandwidth, also increase the Time-on-Air (ToA), which in turn increases the channel usage, ultimately impacting the collision probability [9]. After each transmission, the LORAWAN device opens two receive windows, with duration $T_{r x 1 w}$ and $T_{r x 2 w}$, for downlink messages. Moreover, since LoRA is a type of frequency modulation, it presents the capture effect [24], enabling the receiver to decode the strongest signal when there is a collision, provided that this signal is at least $\theta \mathrm{dB}$ above the interference.

Finally, Table I presents the sensibility, signal-to-noise ratio (SNR) thresholds, and ToA and receive windows time for each SF. It shows that the increase of the SF nearly halves SNR $(-3 \mathrm{~dB})$. Note that all times are longer with higher SF.

\section{SySTEM MODEL}

Similar to [11], consider a circular region with radius $R$ meters and area $V=\pi R^{2}$, where, on average, $\bar{N}$ end-devices are uniformly deployed at random. The positioning of such devices is described by six Poisson Point Processes (PPP) $\Phi_{j}$, one for each $\mathrm{SF} j$, with density $\rho_{j}>0$ in $V$ and 0 otherwise, where $\bar{N}=\rho V$. The distance from $i$-th device to the gateway at the origin is $d_{i}$ meters. Devices transmit using unslottedALOHA. All nodes run the same application, while the ratio of time they transmit in an interval is the activity factor $p_{j}=\frac{t_{j}}{P}$, where $P$ is the average message period, i.e., the time between transmissions, and $t_{j}$ is the time-on-air for $\mathrm{SF} j$, according to Table I. The activity factor vector is $p=\left[p_{7}, \ldots, p_{12}\right]$, each element being the activity factor for a SF. If nodes transmit a given amount of messages per day, due to different ToA per SF, nodes using SF12 would have a larger activity factor than nodes using SF7. Note that the activity factor should respect the regional duty cycle restriction, which is $1 \%$ for the most used configurations in Europe. All devices use the same bandwidth $B \mathrm{~Hz}$ and the same total transmit power $\mathcal{P}_{t}$.

The model considers both path loss attenuation, $g\left(d_{i}\right)$, and Rayleigh fading, $h_{i}$. We use an empirical path loss model of an indoor industrial environment in the sub-GHz band [25], $g\left(d_{i}\right)=\mathrm{PL}_{0}^{-1}\left(\frac{d_{i}}{d_{0}}\right)^{-\eta}$, where $\eta$ is the path loss exponent 
and $\mathrm{PL}_{0}$ is the loss at the reference distance $d_{0}$ meters. Given a signal $s_{1}$ transmitted by a LoRA end-device, the received signal at the gateway, $r_{1}$, is the sum of the attenuated transmitted signal, interference and noise,

$$
r_{1}=\sqrt{\mathcal{P}_{t} g\left(d_{1}\right)} h_{1} s_{1}+\sum_{k \in \Phi_{j}} \sqrt{\mathcal{P}_{t} g\left(d_{k}\right)} h_{k} s_{k}+w,
$$

where $k$, in the summation, iterates over the active nodes indicated by the PPP, and $w$ is the additive white Gaussian noise with zero mean and variance $\mathcal{N}=-174+\mathrm{NF}+$ $10 \log (B) \mathrm{dBm}$, while $\mathrm{NF}$ is the receiver noise figure. An outage takes place either if there is no connection between a node and the gateway or if there is a collision.

\section{A. Outage Condition 1: Disconnection}

The disconnection probability is the complement of the connection probability denoted by $H_{1}$. The connection probability depends directly on the distance between a node and the gateway. A node is considered connected if the SNR of the received signal is above the SNR threshold in Table I. The connection probability can be written as

$$
H_{1}=\mathbb{P}\left[\mathrm{SNR} \geq q_{j} \mid d_{1}\right],
$$

where $q_{j}$ is the SNR threshold for $\mathrm{SF} j$, and $d_{1}$ is the distance between that node and the gateway. The node at $d_{1}$ uses $\mathrm{SF} j$. Assuming Rayleigh fading implies exponentially distributed instantaneous SNR because $\left|h_{1}\right|^{2} \sim \exp (1)$. Thus, $H_{1}$ is

$$
H_{1}=\mathbb{P}\left[\left|h_{1}\right|^{2} \geq \frac{\mathcal{N} q_{j}}{\mathcal{P}_{t} g\left(d_{1}\right)} \mid d_{1}\right]=\exp \left(-\frac{\mathcal{N} q_{j}}{\mathcal{P}_{t} g\left(d_{1}\right)}\right) \text {. }
$$

\section{B. Outage Condition 2: Collision}

A collision happens when packets are simultaneously transmitted using the same SF. Due to LoRA capture effect, the receiver recovers a packet if its power is at least $\theta$ times above the interference. We model the capture probability, i.e., the complement of the collision probability, as in [9]. First, we define the Signal-to-Interference Ratio (SIR) of a packet from a node $d_{1}$ meters from the gateway using $\mathrm{SF} j$ as

$$
\mathrm{SIR}=\frac{\mathcal{P}_{t}\left|h_{1}\right|^{2} g\left(d_{1}\right)}{\sum_{k \in \Phi_{j}} \mathcal{P}_{t}\left|h_{k}\right|^{2} g\left(d_{k}\right)}=\frac{\left|h_{1}\right|^{2} d_{1}^{-\eta}}{\sum_{k \in \Phi_{j}}\left|h_{k}\right|^{2} d_{k}^{-\eta}} .
$$

Then, due to the capture effect, the probability of a successful reception in the presence of interference is

$$
\begin{aligned}
Q_{1} & =\mathbb{P}\left[\operatorname{SIR}>\theta \mid d_{1}\right] \\
& =\mathbb{P}\left[\left|h_{1}\right|^{2}>\theta d_{1}^{\eta} \sum_{k \in \Phi_{j}}\left|h_{k}\right|^{2} d_{k}^{-\eta} \mid d_{1}\right] \\
& =\mathbb{E}_{\left|h_{k}\right|^{2}, \Phi}\left[\prod_{k \in \Phi_{j}} \exp \left(-\theta d_{1}^{\eta}\left|h_{k}\right|^{2} d_{k}^{-\eta}\right)\right] .
\end{aligned}
$$

Since $\left|h_{k}\right|^{2}$ is also exponentially distributed as $\left|h_{1}\right|^{2}$, then

$$
Q_{1}=\mathbb{E}_{\Phi_{j}}\left[\prod_{k \in \Phi_{j}} \frac{1}{1+\theta d_{1}^{\eta} d_{k}^{-\eta}}\right]
$$

Following [9], we use the probability generating functional of the product over PPPs, where $\mathbb{E}\left[\prod_{x \in \Phi} f(x)\right]=$ $\exp \left(-\alpha_{j} \int_{R^{2}} 1-f(x) \mathrm{d} x\right)$ with $\alpha_{j}=2 \rho_{j} p_{j}$ as the PPP density and $d_{k}$ converted to polar coordinates. Note that we double $\alpha_{j}$ due to the use of unslotted-ALOHA [26]. Thus

$$
Q_{1}=\exp \left(-4 \pi \rho_{j} p_{j} \int_{0}^{R} \frac{\theta d_{1}^{\eta} d_{k}^{-\eta}}{1+\theta d_{1}^{\eta} d_{k}^{-\eta}} d_{k} \mathrm{~d} d_{k}\right),
$$

where $R$ is the network radius and $p_{j}$ is the activity factor related to the $\mathrm{SF} j$ used by the node at $d_{1}$.

Finally, by using the definition of the Gauss Hypergeometric function ${ }_{2} F_{1}(a, b ; c ; z)$ [27] we have that

$$
Q_{1}=\exp \left[-2 \pi R^{2} \rho_{j} p_{j}{ }_{2} F_{1}\left(1, \frac{2}{\eta} ; 1+\frac{2}{\eta} ;-\frac{R^{\eta}}{\theta d_{1}^{\eta}}\right)\right] .
$$

\section{Coverage Probability}

The coverage probability is the probability that a node can communicate with the gateway. Since the connection probability in (3) and the probability of a successful reception in the presence of interference in (8) are treated as independent probabilities, we lower bound the coverage probability as $H_{1} Q_{1}$ [28]. Therefore, an upper bound to the link outage probability is defined as $\mathcal{O}_{1}=1-H_{1} Q_{1}$.

\section{Previous Time Diversity Schemes}

This section presents two message replication schemes for LPWANs. For the sake of clearness, we will call information message the first uncoded message transmitted inside a period. All the other messages we call redundant messages.

\section{A. Replication Transmission (RT)}

Hoeller et al. [9] considered RT, simple message replication, where a message is sent $m$ times during one period, disregarding any downlink channel acknowledgment. Message replicas are separated in time to respect the time coherence of the channel, ensuring different channel realizations. Notice that the increase of transmissions inside one period affects $Q_{1}$ since it increases the collision probability. $Q_{1}$ takes the number of interfering nodes as a Poisson distributed variable with mean $v_{j}=p_{j} \rho_{j} V$. With $m$ messages sent in each period, i.e., $M=m$ messages in total, the channel usage increases $M$ times. Thus, it is necessary to adjust the mean of the Poisson distribution proportionally, resulting in $v_{M}=M p_{j} \rho_{j} V$. We denote by $Q_{1, M}$ the capture probability $Q_{1}$ with activity factor $M p_{j}$. Note that $Q_{1}=Q_{1,1}$. The link outage probability is

$$
\mathcal{O}_{M}=1-H_{1} Q_{1, M} \text {. }
$$

The probability of failing to decode all $M$ packets is

$$
\mathcal{O}_{\mathrm{RT}}(M)=\left(\mathcal{O}_{M}\right)^{M} \text {. }
$$




\section{B. Coded Transmission (CT)}

The RT scheme is the simplest way to achieve time diversity. However, lower outages can be obtained by replicating coded messages, which are combinations of previous information messages. The receiver decodes the coded messages to recover lost information, provided that a certain amount of messages is successfully received. Marcelis et al. [20] and MontejoSánchez et al. [10] present cases of embedded redundancy, where packets carry both systematic and parity parts. MontejoSánchez et al. [10] shows, however, that embedded schemes tend to be less reliable, less energy efficient, and provide less coverage than independently coded packet transmissions, as is the case of their proposed independent Coded Transmission (CT) scheme.

The central concept of $\mathrm{CT}$ is to combine different messages using linear operations (e.g., XOR) and send them as independent packets in addition to the information messages. In $\mathrm{CT}$, assuming two information messages $\mathrm{A}$ and $\mathrm{B}$ that are independently transmitted, an additional coded message $\mathrm{A} \oplus \mathrm{B}$ is also transmitted as redundancy. If any two of these three messages are successfully received, the decoder can recover both $\mathrm{A}$ and $\mathrm{B}$ information messages.

The CT scheme has one parameter, $n$, which is the number of coded transmissions per information message. If $n=0$ then no replication takes place. For example, when $n=1$, the $k$ th information message is followed by a coded transmission combining the $k$-th and $(k-1)$-th messages. If $n=2$, the $k$-th information message is followed by a coded transmission combining the $k$-th and $(k-1)$-th messages, and a coded transmission combining the $k$-th and $(k-2)$-th messages. Thus, each information packet is followed, within the period, by $n$ redundant packets. In an infinite sequence of transmissions, each message appears in a window of $2 n$ transmissions in coded messages plus the original information one. Theoretically, there is an infinite amount of combinations of received messages allowing for the recovery of a lost information message. To limit the latency, however, the decoding window of each message is restricted to \pm 3 , i.e., from $(k-3)$-th to $(k+3)$-th. This restriction also allows the deduction of the following closed-form outage probability expression of CT which depends on $M=n+1$ [10]:

$\mathcal{O}_{\mathrm{CT}}(n)=\mathcal{O}_{M}^{2 n+1}\left(1+\mathcal{O}_{M}+\mathcal{O}_{M}^{2}-5 \mathcal{O}_{M}^{3}+4 \mathcal{O}_{M}^{4}-\mathcal{O}_{M}^{5}\right)^{2 n}$.

\section{HybRid REPLICATION SCHEME}

The CT scheme presents a significant improvement compared to RT and other embedded redundancy methods [10]. Note that, for RT, increasing the number of replicas has a beneficial impact in the exponent of the outage probability, but also a negative impact in the number of messages, what increases the number of collisions. In CT, the successive replicas are combined before transmission. The use of linear combinations (e.g., XOR operation) of previous messages increases the redundancy with limited cost in terms of spectral efficiency [10]. However, these linear combinations generate dependence on multiple information for the successful decoding of an encoded message, which is harmful for high
Table II. EXPANSION OF POSSIBLE SUCCESSFUL DECODING EVENTS OF HT FROM $k-3$ TO $k+3$ WITH $n=1$.

\begin{tabular}{|c|c|c|c|c|c|c|c|c|c|c|c|c|c|}
\hline \multicolumn{2}{|c|}{$\mathrm{k}-3$} & \multicolumn{2}{c|}{$\mathrm{k}-2$} & \multicolumn{2}{c|}{$\mathrm{k}-1$} & \multicolumn{2}{c|}{$\mathrm{k}$} & \multicolumn{2}{c|}{$\mathrm{k}+1$} & \multicolumn{2}{c|}{$\mathrm{k}+2$} & \multicolumn{2}{c|}{$\mathrm{k}+3$} \\
\hline $\mathcal{M}$ & $\mathcal{R}$ & $\mathcal{M}$ & $\mathcal{R}$ & $\mathcal{M}$ & $\mathcal{R}$ & $\mathcal{M}$ & $\mathcal{R}$ & $\mathcal{M}$ & $\mathcal{R}$ & $\mathcal{M}$ & $\mathcal{R}$ & $\mathcal{M}$ & $\mathcal{R}$ \\
\hline & & & & & & $\mathrm{S}$ & & & & & & & \\
\hline & & & & $\mathrm{S}$ & & $\mathrm{F}$ & $\mathrm{S}$ & & & & & & \\
\hline & & & & & & $\mathrm{F}$ & & $\mathrm{S}$ & $\mathrm{S}$ & & & & \\
\hline & & $\mathrm{S}$ & & $\mathrm{F}$ & $\mathrm{S}$ & $\mathrm{F}$ & $\mathrm{S}$ & & & & & & \\
\hline & & & & & & $\mathrm{F}$ & & $\mathrm{F}$ & $\mathrm{S}$ & $\mathrm{S}$ & $\mathrm{S}$ & & \\
\hline $\mathrm{S}$ & & $\mathrm{F}$ & $\mathrm{S}$ & $\mathrm{F}$ & $\mathrm{S}$ & $\mathrm{F}$ & $\mathrm{S}$ & & & & & & \\
\hline & & & & & & $\mathrm{F}$ & & $\mathrm{F}$ & $\mathrm{S}$ & $\mathrm{F}$ & $\mathrm{S}$ & $\mathrm{S}$ & $\mathrm{S}$ \\
\hline
\end{tabular}

link outage probabilities. Consequently, a Hybrid Transmission (HT) scheme capable of using replicas of uncoded messages and redundancy based on linear combinations with previous messages should outperform RT and CT. The proposed HT scheme allows for the transmission of uncoded redundant messages only, of coded messages only, or a combination of uncoded and coded redundant replications.

The proposed HT scheme has three parameters: $m$ is the number of uncoded message replicas, as in RT; $n$, as in CT, is the number of different coded messages; and $r$ is the number of replicas of each coded message. For instance, when $n=2$, in the CT scheme a node transmits the $k$-th information message followed by two coded messages: $k$-th $\oplus(k-1)$-th and $k$-th $\oplus$ $(k-2)$-th. In HT, with $n=2, m=2$ and $r=3$, for example, a node sends the $k$-th uncoded message twice $(m=2)$, and then sends two differently coded messages $(n=2)$ three times each $(r=3)$. Then, in HT $M=m+n r$ is the number of messages containing either information or redundancy in a period.

Lemma 1: The outage probability for $H T$ with $n=1$ is a function of two independent sets of events with probability

$$
\begin{aligned}
\mathcal{E}_{m, n, r} & =\left(1-\mathcal{O}_{M}^{m}\right)\left(1-\mathcal{O}_{M}^{r}\right)+\mathcal{O}_{M}^{m}\left(1-\mathcal{O}_{M}^{m}\right)\left(1-\mathcal{O}_{M}^{r}\right)^{2} \\
& +\mathcal{O}_{M}^{2 m}\left(1-\mathcal{O}_{M}^{m}\right)\left(1-\mathcal{O}_{M}^{r}\right)^{3} .
\end{aligned}
$$

Proof: Consider Table II, where each cell indicates whether the decoding of a message was successful (S) or failed (F). Each wide column represents a period, going from $(k-3)$ th to $(k+3)$-th. The narrow columns represent packets containing either original messages $(\mathcal{M})$ or coded messages $(\mathcal{R})$. Each $\mathcal{M}$ or $\mathcal{R}$ column represents events already accounting for the repetitions ( $m$ or $r$ ) of either type of message. Thus, an $\mathrm{F}$ on any cell represents an outage event. Therefore, the outage probability of an original message is $\mathbb{P}(\mathcal{M}=\mathrm{F})=\mathcal{O}_{M}^{m}$, and the outage probability of a coded message is $\mathbb{P}(\mathcal{R}=\mathrm{F})=\mathcal{O}_{M}^{r}$. There are two independent sets of events with the same occurrence probability that allow for the decoding of the $k$ th message if the original transmission fails. The first set, which consists of the events that are a linear combination of the $(k-1)$-th message, is denoted $E_{H, 1}$ and highlighted in blue. The second set is formed by the events that are a linear combination of the $(k+1)$-th message, denoted $E_{H, 2}$ and highlighted in green. Using Table II, we can determine the probability of these events as $\mathbb{P}\left[E_{H, 1}\right]=\mathbb{P}\left[E_{H, 2}\right]=\mathcal{E}_{m, n, r}$. Therefore, the outage probability of HT for $n=1$ can be determined as the union of the events in Table II: the original packet transmission (first line in the table) and the two independent sets of events, considering that the original 
transmission fails. Thus,

$$
\begin{aligned}
\mathcal{O}_{\mathrm{HT}}(m, 1, r) & =1-\left[\left(1-\mathcal{O}_{M}^{m}\right)+\mathcal{O}_{M}^{m} \mathbb{P}\left(E_{H, 1} \bigcup E_{H, 2}\right)\right] \\
& =\mathcal{O}_{M}^{m}-\mathcal{O}_{M}^{m}\left(2 \mathcal{E}_{1, m, r}-\mathcal{E}_{1, m, r}^{2}\right) \\
& =\mathcal{O}_{M}^{m}\left(\mathcal{O}_{M}^{3 m}+\mathcal{O}_{M}^{r}+\mathcal{O}_{M}^{m+r}-2 \mathcal{O}_{M}^{2(m+r)}\right. \\
& -\mathcal{O}_{M}^{3(m+r)}+\mathcal{O}_{M}^{2 m+r}-3 \mathcal{O}_{M}^{3 m+r}-\mathcal{O}_{M}^{m+2 r} \\
& \left.+3 \mathcal{O}_{M}^{3 m+2 r}+\mathcal{O}_{M}^{2 m+3 r}\right)^{2} .
\end{aligned}
$$

Lemma 2: There are $2 n$ independent sets of events, where

$$
\mathbb{P}\left(E_{H, j}\right)=\mathcal{E}_{m, n, r}, \forall j \in\{1, \ldots, 2 n\} .
$$

Proof: Each independent set of events is related to a linear combination of a message. When $n=1$, we have the events that are a linear combination of the $(k-1)$-th and $(k+1)$-th messages. With $n=2$, we also have the events related to the $(k-2)$-th and $(k+2)$-th messages. This set expansion follows as we have sets from the $(k-n)$-th to the $(k+n)$-th messages, totaling $2 n$ independent sets of events.

Theorem 1: The outage probability of $H T$ is

$$
\mathcal{O}_{\mathrm{HT}}(m, n, r)=\mathcal{O}_{M}^{m(2 n+1)}\left(F_{m, n, r}\right)^{2 n},
$$

where

$$
\begin{aligned}
F_{m, n, r} & =\mathcal{O}_{M}^{2 m}+\left(1-\mathcal{O}_{M}^{m}\right)\left(\mathcal{O}_{M}^{m+3 r}-\mathcal{O}_{M}^{2 r}-3 \mathcal{O}_{M}^{m+2 r}\right) \\
& +\mathcal{O}_{M}^{r}\left(1+\mathcal{O}_{M}^{-m}+\mathcal{O}_{M}^{m}-3 \mathcal{O}_{M}^{2 m}\right) .
\end{aligned}
$$

Proof: From Lemma 2, there are $2 n$ independent sets of events, while from the proof of Lemma 1, the outage probability of HT depends on the union of the $2 n$ events. Thus,

$$
\mathcal{O}_{\mathrm{HT}}(m, n, r)=1-\left[\left(1-\mathcal{O}_{M}^{m}\right)+\mathcal{O}_{M}^{m} \mathbb{P}\left(\bigcup_{j=1}^{2 n} E_{H, j}\right)\right] .
$$

Considering the probability of the union of sets of events of the last term, there is an outage only when all $2 n E_{H, j}$ events fail. Thus, we write this success probability as its complement

$$
\mathbb{P}\left(\bigcup_{j=1}^{2 n} E_{H, j}\right)=1-\left(1-\mathcal{E}_{m, n, r}\right)^{2 n}
$$

Applying (12) and (18) to (17) and simplifying yield (15).

Note that if $n=0$ then there is no coding and only simple replicas are sent, making $r$ meaningless. Thus, when $n=0$ (15) reduces to (10), leading to Corollary 1.1.

Corollary 1.1: The HT scheme is a generalization of $R T$ since $\mathcal{O}_{\mathrm{HT}}(m, 0, r)=\mathcal{O}_{R T}(m)$.

When $m=r=1 \mathrm{HT}$ transmits the information message and $n$ coded messages, the same idea as CT, so that (15) reduces to (11), leading to Corollary 1.2.

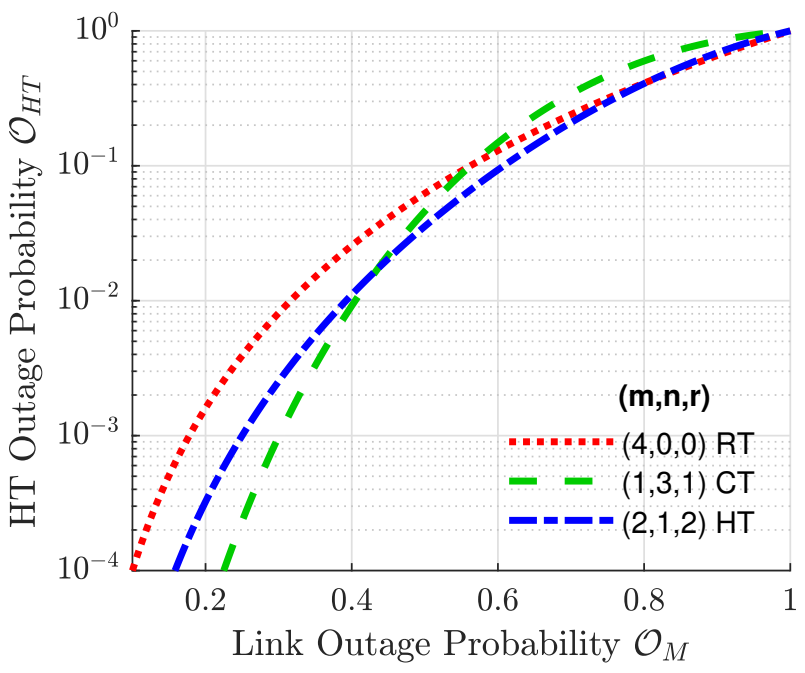

Figure 1. Final outage probability (after replication) versus link outage probability (before replication) for different HT configurations with $M=4$.

Corollary 1.2: The HT scheme is a generalization of $C T$ since $\mathcal{O}_{\mathrm{HT}}(1, n, 1)=\mathcal{O}_{C T}(n)$.

Theorem 2: The outage probability of HT is never larger than that of $R T$ and $C T$, i.e.,

$$
\mathcal{O}_{\mathrm{HT}}(m, n, r) \leq \mathcal{O}_{\mathrm{CT}}(n), \mathcal{O}_{\mathrm{RT}}(m) .
$$

Proof: The proof comes from Corollaries 1.1 and 1.2.

Figure 1 illustrates Theorem 2, showing the final outage probability (after decoding the replications) versus the link outage probability (before decoding the replications), for three possible configurations of the HT scheme with $M=4$, including those equivalent to CT and RT. In this example, the CT-specific configuration performs better for low link outage probability (below $\approx 0.4$ ), and the RT-specific configuration performs slightly better for link outage probabilities very close to 1 . For all other values of $\mathcal{O}_{M}$, the HT-exclusive configuration outperforms the others. Notice that HT is, thus, always better than or equivalent to RT and CT, since it is possible to set HT to mimic the other schemes.

Regarding implementation, devices do not implement any optimization routine apart from acquiring and transmitting data. We should only include simple XOR operations and some buffers at each end-device with size depending on parameter $n$. The network server is in charge of all processing with total control over the network, i.e., it has information on all active devices, including their SF and configuration of the replication scheme $(m, n, r)$. The network server should configure the devices in the join procedure or reconfigure through downlink MAC commands if it deems necessary. It is an implementation choice if the configuration information is in the header or payload as a new message type. However, both cases provide a minimum increase in the message length. For example, with 8 extra bits in a header it is possible to store information about $m$ and $r$ varying from 1 to 8 , and $n$ from 1 to 4 , covering a huge set of different HT configurations. 


\section{A. Average Number of Devices}

As stated in [5], the number of devices is a key requirement of Industrial IoT applications. However, it is also important to cover these devices with adequate reliability levels. We propose to find the maximum number of users that guarantees the minimum reliability level $\mathcal{T}$. To do so, we must ensure that all devices in the network are above $\mathcal{T}$. Thus, we evaluate this metric considering the worst case position in the network, i.e., at the border $\left(d_{1}=R\right)$. From (8), considering $d_{1}=R$ and the activity factor increased by $M$, we have that

$$
Q_{M}=\exp \left[-2 \pi R^{2} \rho_{j} M p_{j}{ }_{2} F_{1}\left(1, \frac{2}{\eta} ; 1+\frac{2}{\eta} ;-\frac{1}{\theta}\right)\right] .
$$

Isolating the node density,

$$
\rho_{j}=-\frac{\ln \left(\frac{1-\mathcal{O}_{M}}{H_{1}}\right)}{2 \pi R^{2} M p_{j}{ }_{2} F_{1}\left(1, \frac{2}{\eta} ; 1+\frac{2}{\eta} ;-\frac{1}{\theta}\right)},
$$

where we have $Q_{M}=\frac{1-\mathcal{O}_{M}}{H_{1}}$, recalling that $O_{M}$ is the link outage probability and $H_{1}$ is the connection probability. Note that since we are investigating the outage at a fixed distance, the connection probability $H_{1}$ is a constant, irrespective of $\rho$. Finally, since the disk total area is $V=\pi R^{2}$ and the average number of nodes is $\bar{N}=\rho V$, we have that

$$
\bar{N}_{j}=-\frac{\ln \left(\frac{1-\mathcal{O}_{M}}{H_{1}}\right)}{2 M p_{j}{ }_{2} F_{1}\left(1, \frac{2}{\eta} ; 1+\frac{2}{\eta} ;-\frac{1}{\theta}\right)} .
$$

Note that $\bar{N}_{j}$ denotes the number of devices using $\mathrm{SF} j$, while $\bar{N}$ is the total number of devices in the network. Given (22) and the outage expressions of the replication schemes, we aim to find the maximum number of nodes supported by the network that guarantees a minimum reliability level. To do so, we need do invert all the equations and find the configuration that minimizes the outage, and thus maximize $\bar{N}$. Since CT and HT equations are quite complicated, we evaluate them numerically enumerating all possible configurations up to a maximum number of message copies $(M)$.

\section{B. Energy Consumption Model}

The lifetime of a device battery is an important concern for Industrial IoT applications. SF plays an important role in LORAWAN energy consumption. Besides the usual impact of transmission power, transmission period, and payload length, SF increases signal length and therefore greatly increases energy expenditure because higher SFs present lower data rates, extending transmission time and, thus consuming more energy. When implementing the presented replication schemes, we expect a trade-off between reliability and battery lifetime.

To quantify the impact of the replication schemes, we use an energy consumption model for unacknowledged LoRAWAN presented in [22], which considers the energy consumption and time duration of 11 operating states of a LORAWAN device.
Table III. ENERGY CONSUMPTION STATES OF LORAWAN [22]

\begin{tabular}{llllll}
\hline \multirow{2}{*}{ State } & \multirow{2}{*}{ Description } & Duration & \multicolumn{3}{c}{ Current } \\
\cline { 3 - 6 } & & Variable & Value $(\mathbf{m s})$ & Variable & Value $(\mathbf{m A})$ \\
\hline 1 & Wake up & $T_{w u}$ & 168.2 & $I_{w u}$ & 22.1 \\
2 & Radio preparation & $T_{p r e}$ & 83.8 & $I_{p r e}$ & 13.3 \\
3 & Transmission & $t$ & Table I & $I_{t x}$ & 83.0 \\
4 & Radio off & $T_{o f f}$ & 147.4 & $I_{o f f}$ & 13.2 \\
5 & Postprocessing & $T_{p o s t}$ & 268.0 & $I_{p o s t}$ & 21.0 \\
6 & Turn off sequence & $T_{s e q}$ & 38.6 & $I_{s e q}$ & 13.3 \\
7 & Wait 1st window & $T_{w 1 w}$ & 983.3 & $I_{1 w}$ & 27.0 \\
8 & 1st receive window & $T_{r x 1 w}$ & Table I & $I_{r x 1 w}$ & 38.1 \\
9 & Wait 2nd window & $T_{w 2 w}$ & $1-T_{r x 1 w}$ & $I_{2 w}$ & 27.1 \\
10 & 2nd receive window & $T_{r x 2 w}$ & Table I & $I_{r x 2 w}$ & 35.0 \\
11 & Sleep & $T_{s l e e p}$ & $(24)$ & $I_{\text {sleep }}$ & $45 \times 10^{-3}$ \\
\hline
\end{tabular}

The average current consumption of a device as a function of time spent in each state is

$$
I_{\text {avg }}=\frac{M}{P}\left(\sum_{i=1}^{N_{\text {states }}-1} T_{i} I_{i}+T_{\text {sleep }} I_{\text {sleep }}\right),
$$

where $M$ is the number of message copies inside a period, $N_{\text {states }}=11, T_{i}$ and $I_{i}$ are respectively the duration and current consumption of state $i$ in Table III and

$$
T_{\text {sleep }}=P-M \sum_{i=1}^{N_{\text {states }}-1} T_{i}
$$

Moreover, we calculate the theoretical lifetime of a batteryoperated device as

$$
T_{\text {lifetime }}=\frac{C_{\text {battery }}}{I_{\text {avg }}},
$$

where $C_{\text {battery }}$ is the battery capacity.

From this model, we see that a considerable amount of energy is spent in the downlink receive windows the device opens after each transmission - in the case of replications, the number of receive windows increases with $M$. Note that unsynchronized Class A nodes must open the receive window for its entire duration, even if it does not detect any downlink message. On top of that, as indicated in [29], the excessive use of LORAWAN downlink can severely worsen network performance. Based on the above facts, we propose that devices only open receive windows when transmitting the last message, avoiding the excessive receive windows when sending redundant replications. With this, the new average current consumption $I_{a v g 2}$ is

$$
I_{\text {avg } 2}=\frac{M}{P}\left(\sum_{i=1}^{N_{\text {states }}-5} T_{i} I_{i}+\sum_{i=7}^{N_{\text {states }}-1} T_{i} I_{i}+T_{\text {sleep } 2} I_{\text {sleep }}\right) \text {, }
$$

where $T_{\text {sleep } 2}$ is the new sleep duration

$$
T_{\text {sleep } 2}=P-M \sum_{i=1}^{N_{\text {states }}-5} T_{i}-\sum_{i=7}^{N_{\text {states }}-1} T_{i} .
$$

Note that the Sleep state is a low power mode, consuming around 1000 times less energy than the other states. 


\section{NUMERICAL RESUltS}

We evaluate the proposed scheme in terms of success probability, energy consumption, and the maximum number of users while meeting a certain reliability target. We compare HT with the other replication schemes RT and CT.

We parameterize the path loss with the empirical data from [25], i.e., path loss exponent $\eta=3.51$, path loss reference $P L_{0}=55.05 \mathrm{~dB}$, and reference distance $d_{0}=15$ meters. We also consider network radius $R=200$ meters as proposed by [30], SIR level $\theta=1 \mathrm{~dB}$ as measured in [12], [31], and total transmit power $\mathcal{P}_{t}=11 \mathrm{dBm}$ as considered by [22]. The LORA transceiver uses bandwidth $B=125 \mathrm{kHz}$ and receiver noise figure $\mathrm{NF}=6 \mathrm{~dB}$. We assume that all devices run the same application with one information message every 10 minutes ( $P=600$ seconds), which means 144 messages per day on average, resulting on activity factors $\{69,120,241,413,826,1652\} \times 10^{-6}$ according to the respective $\mathrm{SF}\{7-12\}$. We consider reliability targets of $\mathcal{T} \in\{0.99,0.999\}$ and restrict the search space of the optimization procedure to $1 \leq M \leq 10$ message copies per period. Note that we limited SF12 up to 6 message copies to adhere to regional duty cycle constraints of $1 \%$. We consider a battery capacity $C_{\text {battery }}=2400 \mathrm{mAh}$, what is typical of AA batteries. Finally, we call DT (Direct Transmission) the baseline scenario with no replication scheme, as in [10], and $\mathrm{HT}^{*}$ the HT scheme with number of messages copies $M$ restrained to at maximum the same number as $\mathrm{CT}$, therefore ensuring a $\mathrm{HT}^{*}$ configuration using the same amount of resources as $\mathrm{CT}$.

Figure 2 compares the outage probability using SF7 for different $\bar{N}_{7}$. We consider a single SF because including them into one curve would cause confusion. Nevertheless, similar plots for other SFs present the same tendency. Also, the numbers next to the curves represent the message copies $(M)$ used to achieve the results in that area. First, we see that any replication scheme performs significantly better than DT. We also see that optimal RT requires more message copies and still has a higher outage than the other schemes. Optimal CT is the scheme that requires fewer message copies and outperforms RT. Optimal HT outperforms all other schemes at the cost of more messages compared to CT. However, optimal HT* can still outperform CT using the same amount of resources.

Figure 3 shows the average number of users in a network to maintain a target success probability $\mathcal{T}$ for different optimized schemes. The parameters RT and CT are detailed in Table IV, while HT parameters are in Table V. Again, we see that HT outperforms the other schemes at the expense of some more message copies. However, $\mathrm{HT}^{*}$ still has better results than $\mathrm{CT}$ using the same resources. Again we see that DT has the worst performance. With higher reliability, $\mathcal{T}=0.999$, we see that the difference from CT and $\mathrm{HT}^{*}$ to $\mathrm{HT}$ is smaller than for $\mathcal{T}=0.99$. This happens because with higher reliability levels, CT tends to perform better, as Figure 1 showed.

Figure 4 presents battery lifetime as a function of message copies for SFs 7 and 12, considering regular LoRAWAN and the proposed adapted LORAWAN with less receive windows. Increasing the number of messages greatly impacts the battery

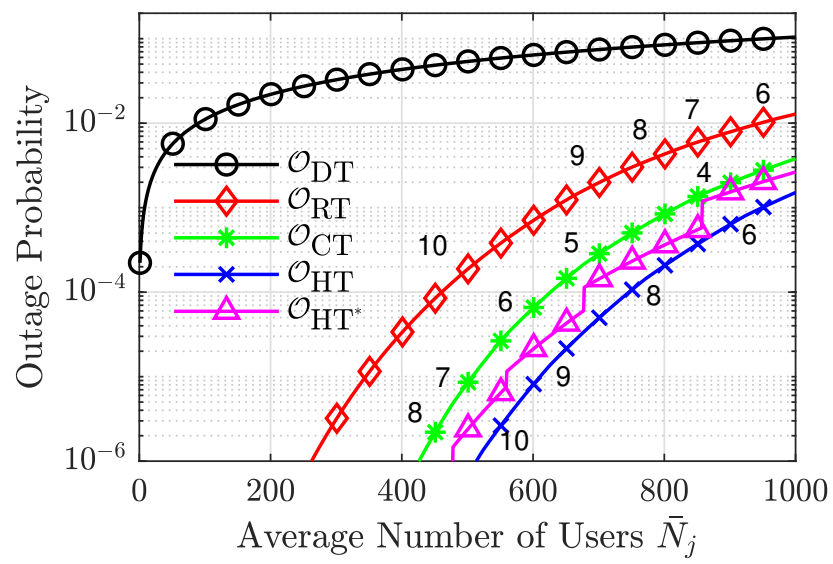

Figure 2. Outage probability of SF7 for each scheme using optimal configuration for different number of users $\bar{N}_{7}$.

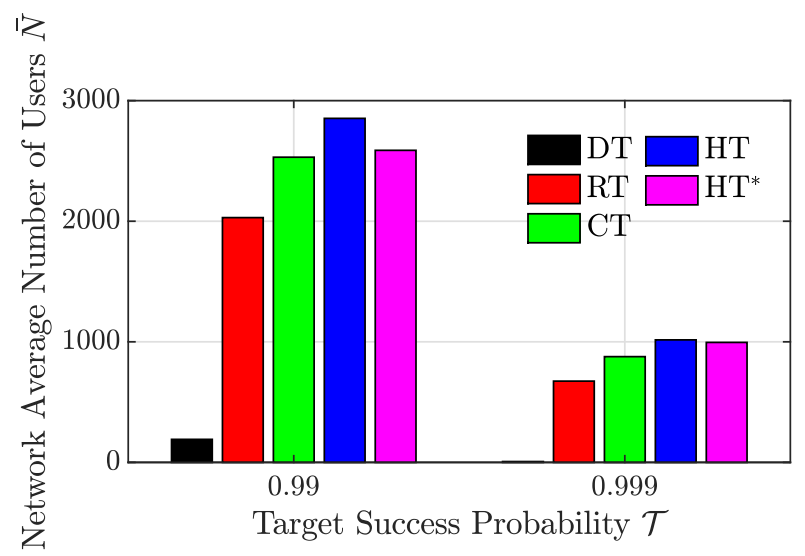

Figure 3. Average number of nodes $\bar{N}$ for each scheme and reliability targets $\mathcal{T} \in\{0.99,0.999\}$.

lifetime, but the impact reduces with the proposed protocol modification. Also, devices using SF7 tend to have higher battery lifetime than SF12, due to SF12 longer ToA.

\section{FINAL COMMENTS}

This paper proposed HT, a coded replication scheme suitable for LORAWAN, which is a generalization of RT and CT. We presented a detailed analysis in terms of outage probability and the number of devices, considering both connection and collision probabilities, showing that RT and CT never outperform HT. The superiority of HT increases with the reliability target, making it suitable for industrial applications. Moreover, HT has a larger set of configuration parameters than RT and CT, being more flexible and becoming able to better adapt to different deployments and requirements. As a future work, we plan to test the proposed scheme in a testbed.

\section{REFERENCES}

[1] M. Centenaro, L. Vangelista, A. Zanella, and M. Zorzi, "Long-range communications in unlicensed bands: the rising stars in the IoT and smart city scenarios," IEEE Wireless Commun., vol. 23, no. 5, pp. 6067, Oct 2016. 


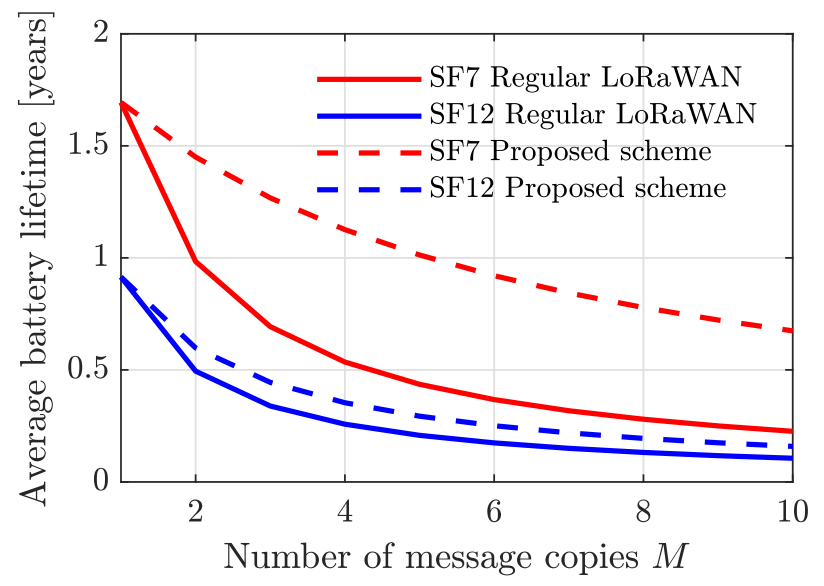

Figure 4. Average device lifetime using SF7 and SF12 for default LORAWAN and the proposed modified protocol.

Table IV. RT AND CT OPTIMAL NUMBER OF MESSAGE COPIES $M$ FOR EACH RELIABILITY TARGET AND SPREADING FACTOR.

\begin{tabular}{lc|c|c|c|c|c|c}
\hline Scheme & $\mathcal{T}$ & SF7 & SF8 & SF9 & SF10 & SF11 & SF12 \\
\hline RT & 0.99 & 7 & 7 & 7 & 7 & 6 & 6 \\
& 0.999 & 10 & 10 & 10 & 9 & 9 & 6 \\
\hline \multirow{2}{*}{ CT } & 0.99 & 3 & 3 & 3 & 3 & 3 & 3 \\
& 0.999 & 5 & 5 & 5 & 5 & 5 & 5 \\
\hline
\end{tabular}

[2] M. de Castro Tomé, P. H. J. Nardelli, and H. Alves, "Long-range low-power wireless networks and sampling strategies in electricity metering," IEEE trans. on Ind. Elect., vol. 66, no. 2, pp. 1629-1637, Feb 2019.

[3] U. Raza, P. Kulkarni, and M. Sooriyabandara, "Low power wide area networks: An overview," IEEE Commun. Surv. Tut., vol. 19, no. 2, pp. 855-873, Apr 2017.

[4] M. Lauridsen, H. Nguyen, B. Vejlgaard, I. Z. Kovacs, P. Mogensen, and M. Sorensen, "Coverage comparison of GPRS, NB-IoT, LORA, and SIGFOX in a $7800 \mathrm{~km}^{2}$ area," in IEEE 85th Vehicular Technology Conference (VTC Spring), June 2017, pp. 1-5.

[5] R. Candell, M. Kashef, Y. Liu, K. B. Lee, and S. Foufou, "Industrial wireless systems guidelines: Practical considerations and deployment life cycle," IEEE Ind. Elect. Mag., vol. 12, no. 4, pp. 6-17, Dec 2018.

[6] LORAWAN 1.1 Specification, LoRA Alliance, Oct. 2017.

[7] AN120.22 LoRA Modulation Basics, Semtech Coorporation, Mar 2015.

[8] LoRA Alliance. LoRAWAN vertical markets. [Online]. Available: https://lora-alliance.org/lorawan-vertical-markets [Accessed on: 201911-05]

[9] A. Hoeller, R. D. Souza, O. L. A. López, H. Alves, M. de Noronha Neto, and G. Brante, "Analysis and performance optimization of LORA networks with time and antenna diversity," IEEE Access, vol. 6, pp. $32820-32829,2018$

[10] S. Montejo-Sánchez, C. A. Azurdia-Meza, R. D. Souza, E. M. G. Fernandez, I. Soto, and A. Hoeller, "Coded redundant message transmission schemes for low-power wide area IoT applications," IEEE Wireless Commun. Lett., vol. 8, no. 2, pp. 584-587, April 2019.

[11] O. Georgiou and U. Raza, "Low power wide area network analysis: Can LORA scale?" IEEE Wireless Commun. Lett., vol. 6, no. 2, pp. 162-165, Apr 2017.

[12] A. Mahmood, E. Sisinni, L. Guntupalli, R. Rondón, S. A. Hassan, and M. Gidlund, "Scalability analysis of a LoRA network under imperfect orthogonality," IEEE trans. on Ind. Inf., vol. 15, no. 3, pp. 1425-1436, March 2019.
Table V. HT AND HT* OPTIMAL CONFIGURATION FOR EACH RELIABILITY TARGET AND SF.

\begin{tabular}{c|cc|cccc|cccc}
\hline \multirow{2}{*}{ Scheme } & Spreading Factor & \multicolumn{4}{c}{ SF7-SF11 } & \multicolumn{4}{c}{ SF12 } \\
\cline { 2 - 10 } & & Parameter & $m$ & $n$ & $r$ & $M$ & $m$ & $n$ & $r$ & $M$ \\
\hline \multirow{2}{*}{ HT } & $\mathcal{T}$ & $\mathbf{0 . 9 9}$ & 2 & 1 & 3 & 5 & 2 & 1 & 3 & 5 \\
& $\mathbf{0 . 9 9 9}$ & 2 & 1 & 4 & 6 & 2 & 1 & 3 & 5 \\
\hline \multirow{2}{*}{ HT $^{*}$} & $\mathcal{T}$ & $\mathbf{0 . 9 9}$ & 1 & 1 & 2 & 3 & 1 & 1 & 2 & 3 \\
& & $\mathbf{0 . 9 9 9}$ & 2 & 1 & 3 & 5 & 2 & 1 & 3 & 5 \\
\hline
\end{tabular}

[13] K. Mikhaylov, J. Petaejaejaervi, and T. Haenninen, "Analysis of capacity and scalability of the LORA low power wide area network technology," in European Wireless, May 2016, pp. 1-6.

[14] P. Sommer, Y. Maret, and D. Dzung, "Low-power wide-area networks for industrial sensing applications," in IEEE Int. Conf. on Ind. Internet (ICII), Oct 2018, pp. 23-32.

[15] M. Rizzi, P. Ferrari, A. Flammini, E. Sisinni, and M. Gidlund, "Using LORA for industrial wireless networks," in IEEE Int. Works. on Factory Commun. Syst. (WFCS), May 2017, pp. 1-4.

[16] M. I. Muzammir, H. Z. Abidin, S. A. C. Abdullah, and F. H. K. Zaman, "Performance analysis of LORAWAN for indoor application," in IEEE Symp. on Comp. App. Ind. Elect. (ISCAIE), April 2019, pp. 156-159.

[17] J. Haxhibeqiri, A. Karaagac, F. Van den Abeele, W. Joseph, I. Moerman, and J. Hoebeke, "LORA indoor coverage and performance in an industrial environment: Case study," in IEEE Int. Conf. on Emerging Tech. and Fact. Aut. (ETFA), Sep. 2017, pp. 1-8.

[18] J. Lentz, S. Hill, B. Schott, M. Bal, and R. Abrishambaf, "Industrial monitoring and troubleshooting based on LORA communication technology," in IECON - Conf. of the IEEE Ind. Elect. Society, Oct 2018, pp. 3852-3857.

[19] Y. Mo, M.-T. Do, C. Goursaud, and J.-M. Gorce, "Optimization of the predefined number of replications in a ultra narrow band based IoT network," in Wireless Days (WD), Mar 2016.

[20] P. J. Marcelis, V. S. Rao, and R. V. Prasad, "Dare: Data recovery through application layer coding for LORAWAN," in IEEE/ACM Int. Conf. on Internet-of-Things Design and Implem. (IoTDI), April 2017, pp. 97108.

[21] M. Sandell and U. Raza, "Application layer coding for IoT: Benefits, limitations, and implementation aspects," IEEE Syst. J., vol. 13, no. 1, pp. 554-561, March 2019.

[22] L. Casals, B. Mir, R. Vidal, and C. Gomez, "Modeling the energy performance of LoRAWAN," Sensors, vol. 17, no. 10, 2017.

[23] A. Goldsmith, Wireless Communications. New York, NY, USA: Cambridge University Press, 2005.

[24] M. Bor, U. Roedig, T. Voigt, and J. M. Alonso, "Do LoRA low-power wide-area networks scale?" in 19th ACM Int. Conf. on Modeling, Anal., and Simulation of Wireless Mobile Syst. (MSWiM), New York, USA, 2016, pp. 59-67.

[25] E. Tanghe, W. Joseph, L. Verloock, L. Martens, H. Capoen, K. V. Herwegen, and W. Vantomme, "The industrial indoor channel: largescale and temporal fading at 900, 2400, and $5200 \mathrm{MHz}$, IEEE trans. on Wireless Commun., vol. 7, no. 7, pp. 2740-2751, July 2008.

[26] M. Berioli, G. Cocco, G. Liva, and A. Munari, "Modern random access protocols," Foundations and Trends in Networking, vol. 10, no. 4, pp. 317-446, 2016.

[27] A. B. O. Daalhuis, "Hypergeometric function," in NIST Handbook of Mathematical Functions, 1st ed., F. W. J. Olver, D. W. Lozier, R. F. Boisvert, and C. W. Clark, Eds. New York, USA: Cambridge University Press, 2010, ch. 15, pp. 383-402.

[28] L. Beltramelli, A. Mahmood, M. Gidlund, P. Österberg, and U. Jennehag, "Interference modelling in a multi-cell LoRA system," in 14th International Conference on Wireless and Mobile Computing, Networking and Communications (WiMob), Oct 2018, pp. 1-8. 
[29] M. Centenaro, L. Vangelista, and R. Kohno, "On the impact of downlink feedback on LORA performance," in IEEE Int. Symp. on Pers., Ind. and Mob. Radio Commun. (PIMRC), Oct 2017, pp. 1-6.

[30] M. Luvisotto, F. Tramarin, L. Vangelista, and S. Vitturi, "On the use of LORAWAN for indoor industrial iot applications," Wireless Commun. and Mobile Comput., vol. 2018, p. 11, 2018.

[31] D. Croce, M. Gucciardo, S. Mangione, G. Santaromita, and I. Tinnirello, "Impact of LoRA imperfect orthogonality: Analysis of link-level performance," IEEE Commun. Lett., vol. 22, no. 4, pp. 796-799, April 2018. 